\title{
Just Add Water: A New Fluorous Capping Reagent for Facile Purification of Peptides Synthesized on the Solid Phase
}

Vittorio Montanari and Krishna Kumar*

Department of Chemistry, Tufts University, Medford, MA 02155-5813

J. Am. Chem. Soc.

\section{Supplementary Information}

\section{Peptide Synthesis}

Peptides were prepared using the $N$-tert-butyloxycarbonyl ( $t$-Boc) amino acid derivatives for Merrifield solid-phase synthesis (MBHA resin) using the in-situ neutralization protocol. ${ }^{1} N-\alpha$-Boc- $\alpha$-S-amino acids were used with standard side chain protecting groups.

At the points of intentional incomplete couplings, $0.8-0.9$ equivalents of Boc-amino acid (0.12-0.135 mmol for a $0.15 \mathrm{mmol}$ scale synthesis), $0.72-0.81$ eq. HBTU, and 3.5 eq. DIEA were used.

Peptides were cleaved from the resin using high HF conditions (90\% anhydrous $\mathrm{HF} / 10 \%$ anisole at $0{ }^{\circ} \mathrm{C}$ for 2 hours). The crude reaction mixture after the cleavage reaction was washed with cold $\mathrm{Et}_{2} \mathrm{O}$. Peptides were extracted with $5 \times 15 \mathrm{~mL} 10 \% \mathrm{AcOH}$, followed by $5 \times 15 \mathrm{~mL} 50 \% \mathrm{CH}_{3} \mathrm{CN}$ in water (containing $0.1 \% \mathrm{CF}_{3} \mathrm{COOH}$ ).

The $\mathrm{AcOH}$ and $\mathrm{CH}_{3} \mathrm{CN}$ extracts were separately reduced to small volume using a rotary evaporator, the residues taken up in water and lyophilized.

\section{Preparation and Characterization of Reagent 1:}

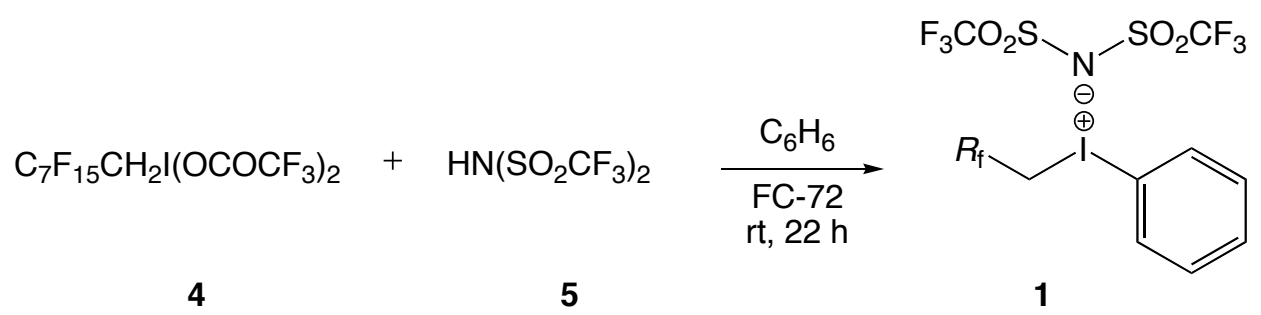

(Bis-trifluoroacetoxy)iodo- $1 \mathrm{H}, 1 \mathrm{H}$-perfluorooctane ${ }^{2}$ (4) $(6.0 \mathrm{~g}, 8.2 \mathrm{mmol})$ was added in one portion to $\left(\mathrm{CF}_{3} \mathrm{SO}_{2}\right)_{2} \mathrm{NH}(\mathbf{5})(3.01 \mathrm{~g}, 10.7 \mathrm{mmol})$. The triflimide $\left(\mathrm{CF}_{3} \mathrm{SO}_{2}\right)_{2} \mathrm{NH}$ should be blanketed with nitrogen because it strongly fumes and deliquesces in the air. The two solids were slurried in a mixture of FC-72 (perfluorohexane, $12 \mathrm{~mL}$ ) and trifluoroacetic 
anhydride $(3 \mathrm{~mL})$. A clear solution formed within 15 minutes, at which point benzene $(1.2 \mathrm{~mL}, 13.3 \mathrm{mmol})$ was added rapidly and the reaction mixture was stirred under nitrogen for $22 \mathrm{hrs}$ at $\mathrm{rt}$. Evaporation of the volatiles and stirring with ice/water produced a white precipitate. This was collected on a glass frit, dried, and further evacuated under $0.1 \mathrm{~mm} \mathrm{Hg}$ yielding $6.12 \mathrm{~g}(85 \%)$ of 1 as a white powder, which was used directly in capping reactions. ${ }^{1} \mathrm{H}$ NMR $\left(\mathrm{CD}_{2} \mathrm{Cl}_{2}, 300 \mathrm{MHz}\right.$, ref. $\left.\mathrm{CHDCl}=5.3 \mathrm{ppm}\right)$ $4.8\left(\mathrm{t}, 2 \mathrm{H}, J=18 \mathrm{~Hz}, \mathrm{CH}_{2} R_{\mathrm{f}}\right), 7.6(\mathrm{~m}, 2 \mathrm{H}), 7.8(\mathrm{~m}, 1 \mathrm{H}), 8.2(\mathrm{~m}, 2 \mathrm{H}) .{ }^{19} \mathrm{~F} \mathrm{NMR}\left(\mathrm{CD}_{2} \mathrm{Cl}_{2}\right.$, $282 \mathrm{MHz}$, ref. $\left.\mathrm{C}_{6} \mathrm{~F}_{6}=-162 \mathrm{ppm}\right)-80.6\left(\mathrm{~s}, 6 \mathrm{~F}, \mathrm{SO}_{2} \mathrm{CF}_{3}\right),-82.0\left(\mathrm{~m}, 3 \mathrm{~F}, \mathrm{CF}_{3}\right),-105.0(\mathrm{~m}$, $\mathrm{CH}_{2} \mathrm{CF}_{2}$ ), -122.1 (4F, br s, two overlapping $\mathrm{CF}_{2}$ ), -122.8 (2F, br s), -123.5 (2F, br s), -127.0 (2F, br s).

\section{Fluorous Capping with Reagent 1}

After the intentional incomplete coupling, the resin was washed with DMF (30 sec flow wash and $5 \times 10 \mathrm{~mL}$ shake wash). The same wash sequence was repeated using $\mathrm{CH}_{2} \mathrm{Cl}_{2}$. Then 2 eq of a solution of 1 in $\mathrm{CH}_{2} \mathrm{Cl}_{2}\left(0.3 \mathrm{mmol}, 260 \mathrm{mg}\right.$ in $5 \mathrm{~mL} \mathrm{CH}_{2} \mathrm{Cl}_{2}$ for a 0.15 mmol scale synthesis) were added and the resin was mildly shaken for $1 \mathrm{~min}$. Then a solution of $2 \mathrm{eq}$ ( $40 \mu \mathrm{L}$ for a $0.15 \mathrm{mmol}$ scale synthesis) of 2,4,6-collidine in $\mathrm{CH}_{2} \mathrm{Cl}_{2}(2$ $\mathrm{mL}$ ) was added and mild shaking continued for $15 \mathrm{~min}$. The resin was washed with $\mathrm{CH}_{2} \mathrm{Cl}_{2}$ and the capping step was repeated. Deprotection was performed with neat TFA after washing with $\mathrm{CH}_{2} \mathrm{Cl}_{2}$ (30 sec flow). After deprotection the resin was again swollen in DMF and the synthesis continued as usual.

\section{Fluoroalkylation of Compound 2}

Compound 2 (H-Tyr-OBut, $119 \mathrm{mg}, 0.5 \mathrm{mmol}$ ), iodonium salt 1 (430 mg, $0.5 \mathrm{mmol}$ ), and 2,4,6-collidine $(66 \mu \mathrm{L}, 0.5 \mathrm{mmol})$ were stirred in $\mathrm{CH}_{2} \mathrm{Cl}_{2}(5 \mathrm{~mL})$ at room temperature for 1 hour (by TLC the reaction was judged complete in $10 \mathrm{~min}$ ). Evaporation to small volume and chromatography $\left(\mathrm{Et}_{2} \mathrm{O} /\right.$ hexanes $\left.1 / 1\right)$ gave $248 \mathrm{mg}(80 \%)$ of $\mathrm{C}_{7} \mathrm{~F}_{15} \mathrm{CH}_{2}-\mathrm{NH}-$ Tyr-OBut as a white powder. ${ }^{1} \mathrm{H}$ NMR $\left(\mathrm{CDCl}_{3}, 300 \mathrm{MHz}\right.$, ref. TMS $\left.=0 \mathrm{ppm}\right) 1.4(\mathrm{~s}, 9$ $\mathrm{H}, \mathrm{Bu} t), 2.8-2.9\left(\mathrm{~m}, 2 \mathrm{H}\right.$, tyrosine $\left.\mathrm{CH}_{2}\right), 3.0-3.5\left(\mathrm{ABX}_{2} \mathrm{~m}, 2 \mathrm{H}, R_{\mathrm{f}} \mathrm{CF}_{2} \mathrm{CH}_{2}\right), 3.5(\mathrm{t}, 1 \mathrm{H}), 6.7$ $(\mathrm{d}, 2 \mathrm{H}), 7.0(\mathrm{~d}, 2 \mathrm{H}) .{ }^{19} \mathrm{~F}$ NMR $\left(\mathrm{CDCl}_{3}, 282 \mathrm{MHz}\right.$, ref. $\left.\mathrm{C}_{6} \mathrm{~F}_{6}=-162 \mathrm{ppm}\right)-81.0(\mathrm{~m}, 3 \mathrm{~F}$, $\mathrm{CF}_{3}$ ), -118.4 (m, $\mathrm{CH}_{2} \mathrm{CF}_{2}$ ), -122.0 (4F, br s, two overlapping $\mathrm{CF}_{2}$ ), -123.0 (2 F, br s), -123.5 (2F, br s), -126.2 (2 F, br s).

H-Tyr-OBut (24 mg, $0.1 \mathrm{mmol}$ ), iodonium salt (173 mg, $0.2 \mathrm{mmol}$ ), and 2,4,6-collidine (27 $\mu \mathrm{L}, 0.2 \mathrm{mmol})$ were stirred in $\mathrm{CH}_{2} \mathrm{Cl}_{2}(3 \mathrm{~mL})$ at room temperature for 15 minutes (a typical capping reaction time), then DIEA $(50 \mu \mathrm{L})$ was added to destroy any remaining iodonium salt. TLC and NMR analysis indicated no other product besides $\mathrm{C}_{7} \mathrm{~F}_{15} \mathrm{CH}_{2}-\mathrm{NH}-$ Tyr-OBut.

Similarly, $\mathrm{C}_{7} \mathrm{~F}_{15} \mathrm{CH}_{2}-\mathrm{NH}-\mathrm{Tyr}-\mathrm{OBu} t(35 \mathrm{mg}, 0.057 \mathrm{mmol})$, iodonium salt (100 mg, 0.115 mmol) and 2,4,6-collidine (16 $\mu \mathrm{L}, 0.12 \mathrm{mmol})$ were stirred in $\mathrm{CH}_{2} \mathrm{Cl}_{2}(2 \mathrm{~mL})$ at room temperature for 30 minutes. TLC indicated only $\mathrm{C}_{7} \mathrm{~F}_{15} \mathrm{CH}_{2}-\mathrm{NH}-\mathrm{Tyr}-\mathrm{OBu} t$ and $\mathrm{PhI}$ as the low polarity components. The reaction was quenched with DIEA $(50 \mu \mathrm{L})$ and the 
solvents evaporated. Chromatography on silica gel $\left(\mathrm{Et}_{2} \mathrm{O} /\right.$ hexanes $\left.1 / 1\right)$ gave pure $\mathrm{C}_{7} \mathrm{~F}_{15} \mathrm{CH}_{2}-\mathrm{NH}-\mathrm{Tyr}-\mathrm{OBut}$ ( $29 \mathrm{mg}, 82 \%$ recovery after flash chromatography).

\section{Control Reaction to demonstrate inertness of $\mathrm{R}_{\mathrm{f}} \mathrm{CH}_{2} \mathrm{NH}$ - to peptide coupling reactions}

A solution of Boc-Ala-OH (66 mg, $0.35 \mathrm{mmol}), \mathrm{HBTU}(118 \mathrm{mg}, 0.31 \mathrm{mmol})$ and DIEA $(90 \mu \mathrm{L}, 0.52 \mathrm{mmol})$ in DMF $(1.5 \mathrm{~mL})$ was stirred for two minutes then transferred to a flask containing monofluoroalkyated compound 3 (60 mg, $0.087 \mathrm{mmol})$. TLC analysis showed only starting material $\left(R_{\mathrm{f}}=0.5\right.$ in hexanes/ $\left.\mathrm{Et}_{2} \mathrm{O} 4 / 1\right)$. After $30 \mathrm{~min}$, the reaction mixture was diluted with $30 \mathrm{~mL}$ of $\mathrm{Et}_{2} \mathrm{O}$ and washed with $2 \times 30 \mathrm{~mL} \mathrm{H}_{2} \mathrm{O}$. The $\mathrm{Et}_{2} \mathrm{O}$ was dried $\left(\mathrm{MgSO}_{4}\right)$ and the solvent evaporated. The residue thus obtained was filtered through silica gel using $\mathrm{Et}_{2} \mathrm{O}$ :hexanes (1:3) to yield $54 \mathrm{mg}(90 \%)$ of unchanged 3 (NMR, TLC). 


\section{Mass Spectrometry (ESI-MS)}

Peptide P1

\begin{tabular}{|c|c|c|c|}
\hline \multicolumn{4}{|c|}{$\begin{array}{l}\text { Peptide P1: Ac-NH-VEA*AID*YI*DA-CONH }{ }_{2} \\
\text { Ac cap with } \mathrm{Ac}_{2} \mathrm{O}\end{array}$} \\
\hline $\begin{array}{l}\text { Fragment } \\
\end{array}$ & $\mathrm{MW}_{\text {calc }}(\mathrm{Da})$ & $\mathrm{MW}_{\text {obsd }}(\mathrm{Da})$ & Sample \\
\hline Ac-NH-VEAAIDYIDA-CONH ${ }_{2}$ & 1119.55 & $1142.55(+\mathrm{Na})$ & 1 \\
\hline Ac-NH-AIDYIDA-CONH 2 & 820.40 & $843.45(+\mathrm{Na})$ & 1 \\
\hline Ac-NH-YIDA-CONH ${ }_{2}$ & 521.25 & $x$ & $x$ \\
\hline Ac-NH-DA-CONH ${ }_{2}$ & 245.10 & $268.18(+\mathrm{Na})$ & 1 \\
\hline \multicolumn{4}{|l|}{$\begin{array}{l}\text { Peptide } \mathbf{P 1} \\
-\mathrm{CH}_{2}-\mathrm{R}_{\mathrm{f}} \text { cap with reagent } \mathbf{1}\end{array}$} \\
\hline $\begin{array}{l}\text { Fragment } \\
\end{array}$ & $\mathrm{MW}_{\text {calc }}(\mathrm{Da})$ & $\mathrm{MW}_{\text {obsd }}(\mathrm{Da})$ & Sample \\
\hline Ac-NH- VEAAIDYIDA-CONH ${ }_{2}$ & 1119.55 & $\begin{array}{l}1120.36 \\
1142.55(+\mathrm{Na})\end{array}$ & 2 \\
\hline $\mathrm{R}_{\mathrm{f}} \mathrm{CH}_{2}-\mathrm{NH}-\mathrm{AIDYIDA}-\mathrm{CONH}_{2}$ & 1160.37 & $\times$ & 2,4 \\
\hline $\mathrm{R}_{\mathrm{f}} \mathrm{CH}_{2}-\mathrm{NH}-\mathrm{YIDA}-\mathrm{CONH}_{2}$ & 861.22 & 862.18 & 2,4 \\
\hline $\mathrm{R}_{\mathrm{f}} \mathrm{CH}_{2}-\mathrm{NH}-\mathrm{DA}-\mathrm{CONH}_{2}$ & 585.07 & $\begin{array}{l}586.09 ; 608.18 \\
(+\mathrm{Na})\end{array}$ & 2,4 \\
\hline
\end{tabular}

Peptide P2

\begin{tabular}{|c|c|c|c|}
\hline \multicolumn{4}{|c|}{$\begin{array}{l}\text { Peptide P2 : Ac-NH-RAV*KVY*ADAA*EDESAEAFALEF-CONH }{ }_{2} \\
\text { Ac cap with } \mathrm{Ac}_{2} \mathrm{O}\end{array}$} \\
\hline Fragment & $\mathrm{MW}_{\text {calc }}(\mathrm{Da})$ & $\mathrm{MW}_{\mathrm{obsd}}(\mathrm{Da})$ & Sample \\
\hline $\begin{array}{l}\text { Ac-NH-RAVKVYADAAEDESAEAFALEF- } \\
\mathrm{CONH}_{2}\end{array}$ & 2442.17 & 1222.64 & 1,3 \\
\hline Ac-NH-KVYADAAEDESAEAFALEF-CONH ${ }_{2}$ & 2115.96 & $1070.55(+\mathrm{Na})$ & 3 \\
\hline Ac-NH-ADAAEDESAEAFALEF-CONH ${ }_{2}$ & 1725.74 & $1748.82(+\mathrm{Na})$ & 3 \\
\hline Ac-NH-EDESAEAFALEF-CONH ${ }_{2}$ & 1397.60 & $1420.73(+\mathrm{Na})$ & 3 \\
\hline \multicolumn{4}{|l|}{$\begin{array}{l}\text { Peptide } \mathbf{P} 2 \\
-\mathrm{CH}_{2}-\mathrm{R}_{\mathrm{f}} \text { cap with reagent } \mathbf{1}\end{array}$} \\
\hline Fragment & $\mathrm{MW}_{\text {calc }}(\mathrm{Da})$ & $\mathrm{MW}_{\text {obsd }}(\mathrm{Da})$ & Sample \\
\hline $\begin{array}{l}\text { Ac-NH-RAVKVYADAAEDESAEAFALEF- } \\
\mathrm{CONH}_{2}\end{array}$ & 2442.17 & 1222.64 & $1,2,3$ \\
\hline $\mathrm{R}_{\mathrm{f}} \mathrm{CH}_{2}-\mathrm{NH}-\mathrm{KVYADAAEDESAEAFALEF-CONH}{ }_{2}$ & 2455.94 & $\times$ & 2 \\
\hline $\mathrm{R}_{\mathrm{f}} \mathrm{CH}_{2}-\mathrm{NH}-\mathrm{ADAAEDESAEAFALEF-CONH}{ }_{2}$ & 2065.71 & $\begin{array}{l}1020.55\left(-\mathrm{H}_{2} \mathrm{O}\right. \\
+\mathrm{Na}) ; 1056.00 \\
(+2 \mathrm{Na})\end{array}$ & 2 \\
\hline $\mathrm{R}_{\mathrm{f}} \mathrm{CH}_{2}-\mathrm{NH}-\mathrm{EDESAEAFALEF}-\mathrm{CONH}_{2}$ & 1737.57 & $\begin{array}{l}1760.54 \\
(+\mathrm{Na}) ; \\
1742.53\left(-\mathrm{H}_{2} \mathrm{O}\right. \\
+\mathrm{Na})\end{array}$ & 2 \\
\hline
\end{tabular}




\begin{tabular}{|c|c|c|c|}
\hline \multicolumn{4}{|l|}{$\begin{array}{l}\text { Peptide P3: PT*GYGS*SSRRAPET } \\
\text { Ac cap with } \mathrm{Ac}_{2} \mathrm{O}\end{array}$} \\
\hline Fragment & $\mathrm{MW}_{\text {calc }}(\mathrm{Da})$ & $\mathrm{MW}_{\mathrm{obsd}}(\mathrm{Da})$ & Sample \\
\hline Ac-NH-PTGYGSSSRRAPET-CONH ${ }_{2}$ & 1505.72 & 1506.73 & 1 \\
\hline Ac-NH-GYGSSSRRAPET-CONH ${ }_{2}$ & 1307.62 & 1308.64 & 1 \\
\hline Ac-NH-SSRRAPET-CONH ${ }_{2}$ & 943.48 & $\begin{array}{l}969.6(+\mathrm{Na}) \\
923.9\left(-\mathrm{H}_{2} \mathrm{O}\right)\end{array}$ & 1 \\
\hline \multicolumn{4}{|l|}{$\begin{array}{l}\text { Peptide } \mathbf{P 3} \\
-\mathrm{CH}_{2}-\mathrm{R}_{\mathrm{f}} \text { cap with reagent } \mathbf{1}\end{array}$} \\
\hline Fragment & $\mathrm{MW}_{\text {calc }}(\mathrm{Da})$ & $\mathrm{MW}_{\text {obsd }}(\mathrm{Da})$ & Sample \\
\hline Ac-NH-PTGYGSSSRRAPET-CONH & 1505.72 & 1506.82 & 1,3 \\
\hline $\mathrm{R}_{\mathrm{f}} \mathrm{CH}_{2}-\mathrm{NH}-\mathrm{GYGSSSRRAPET}-\mathrm{CONH}_{2}$ & 1647.59 & 1648.64 & 1 \\
\hline $\mathrm{R}_{\mathrm{f}} \mathrm{CH}_{2}-\mathrm{NH}-\mathrm{SSRRAPET}-\mathrm{CONH}_{2}$ & 1283.45 & 1284.45 & 1,3 \\
\hline
\end{tabular}

ESI-MS Conditions: Finnigan LTQ, Thermo Electron Corp.; solutions were $\sim 100 \mathrm{nM}$ in $\mathrm{CH}_{3} \mathrm{OH} / 1 \% \mathrm{HCO}_{2} \mathrm{H}$ unless otherwise noted, flow rate: $5 \mu \mathrm{L} / \mathrm{min}$, spray voltage: $5 \mathrm{kV}$, capillary voltage: $40 \mathrm{~V}$.

Sample identities:

(1) Crude obtained from cleavage reaction by resin extraction with $10 \% \mathrm{AcOH}$

(2) Crude obtained from cleavage reaction by resin extraction with $1: 1 \mathrm{CH}_{3} \mathrm{CN}: \mathrm{H}_{2} \mathrm{O}(10 \% \mathrm{AcOH})$

(3) Peak collected from RP-HPLC.

(4) Pellet obtained by centrifugation of sample from (1) and redissolution in $\mathrm{MeOH} / \mathrm{H}_{2} \mathrm{O}$ or $i \mathrm{PrOH} / \mathrm{H}_{2} \mathrm{O}$.

(x) Not observed.

${ }^{1}$ Schnölzer, M.; Alewood, P.; Jones, A.; Alewood.; D, Kent, S. B. Int. J. Pept. Protein Res. 1992, 40, 180-193.

${ }^{2}$ Umemoto, T.; Gotoh, Y. J. Fluorine Chem. 1985, 28, 235-239. 